\title{
Mobile Stroke Unit Reduces Time to Image Acquisition and Reporting
}

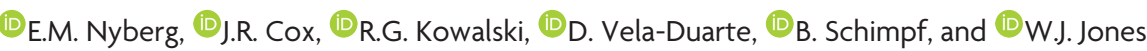

\begin{abstract}
SUMMARY: Timely administration of thrombolytic therapy is critical to maximizing the likelihood of favorable outcomes in patients with acute ischemic stroke. Although emergency medical service activation overall improves the timeliness of acute stroke treatment, the time from emergency medical service dispatch to hospital arrival unavoidably decreases the timeliness of thrombolytic administration. Our mobile stroke unit, a new-generation ambulance with on-board CT scanning capability, reduces key imaging time metrics and facilitates in-the-field delivery of IV thrombolytic therapy.
\end{abstract}

ABBREVIATIONS: EMS = emergency medical service; $M S U$ = mobile stroke unit

A cute ischemic stroke is the fifth leading cause of mortality in the United States and remains the leading cause of morbidity and long-term disability, costing approximately $\$ 33$ billion per year. ${ }^{1}$ The effectiveness of tPA in improving outcomes has been shown to be sensitive to the timeliness of drug delivery, ${ }^{2}$ with a $20 \%$ decrease in the likelihood of a good outcome (defined by a modified Rankin Scale score of 0-2) at 90 days for every 30-minute delay in reperfusion. ${ }^{3}$ Besides decreasing the effectiveness of the drug, delays in care also preclude some patients from receiving thrombolytic therapy when the delays place patients outside the 4.5 -hour treatment window. Currently, only 3\%-5\% of patients with acute ischemic stroke receive thrombolytic therapy, often due to the time-limited treatment window. ${ }^{4}$ Until recently, the time required for prehospital evaluation and transportation via emergency medical service (EMS) has generally been unavoidable. With the introduction of mobile stroke units (MSUs), the time required for transportation and repeat patient evaluation before IV tPA administration can be eliminated.

The purpose of this study was to compare pertinent imaging time metrics for patients imaged in the MSU with those imaged via the conventional pathway through the emergency depart-

Received February 4, 2018; accepted after revision March 27.

From the Departments of Radiology (E.M.N., J.R.C.) and Neurology (R.G.K., D.V.D., B.S., W.J.J.), University of Colorado, Aurora, Colorado.

A previous version of these data was presented at: Annual Meeting of the American Society of Neuroradiology and the Foundation of the ASNR Symposium, April 22-27, 2017; Long Beach, California.

Please address correspondence to Eric M. Nyberg, MD, Department of Radiology, University of Colorado, 12605 E 16th Ave, Aurora, CO 80045; e-mail:

Eric.Nyberg@ucdenver.edu

http://dx.doi.org/10.3174/ajnr.A5673 ment. Specifically, we compared the time of EMS dispatch with the availability of CT images on a PACS and the time of EMS dispatch with the time of a radiology report provided to the neurologist between MSU and conventional pathway groups.

\section{MATERIALS AND METHODS \\ Mobile Stroke Unit}

Our MSU is a new-generation ambulance with an on-board CereTom CT scanner (NeuroLogica, Danvers, Massachusetts). CT images are transferred to the hospital-based PACS system via a Health Insurance Portability and Accountability Act-compliant, 4G network cloud-based system for a radiologist's interpretation. Teleneurology capabilities allow real-time remote examination of the patient by a stroke neurologist. The on-board MSU team includes a stroketrained nurse who can deliver tPA in the field under the direction of the remote stroke neurologist, as well as a CT technologist, paramedic, and an emergency medical technician.

\section{Prehospital Stroke Alerts}

Current stroke guidelines indicate that when an emergency medical technician suspects that a patient is having an acute ischemic stroke on prehospital assessment, that emergency medical technician should contact the receiving emergency department to alert them that the patient is a "prehospital stroke alert." This results in a streamlined patient work-up on arrival to the emergency department. Every other week, if the EMS dispatcher suspects acute ischemic stroke based on the initial call, then our MSU is dispatched simultaneously with the standard EMS service in our region. If on arrival to the scene, the patient is deemed a potential tPA candidate, the MSU assumes control of the patient's transport and care. If the patient is not a tPA candidate, the MSU leaves 
Table 1: Mean times from dispatch to images viewable on PACS and dispatch to report

\begin{tabular}{lccccccc}
\hline & \multicolumn{2}{c}{ MSU } & & \multicolumn{2}{c}{ Control } & & \\
\cline { 2 - 3 } Time (min) & Mean & SD & & Mean & SD & Difference & P Value \\
\hline PACS time & 21 & 5.7 & & 44 & 46.3 & 23 & $<.001$ \\
Report time & 34 & 8.8 & 55 & 47.1 & 21 & $<.001$ \\
\hline
\end{tabular}

Table 2: Group differences in imaging times related to treatment benchmarks

\begin{tabular}{lrrrrrrrr} 
& \multicolumn{3}{c}{ PACS Time } & & \multicolumn{3}{c}{ Report Time } \\
\cline { 2 - 4 } \cline { 6 - 8 } Time & MSU & Control & $\boldsymbol{P}$ Value & & MSU & Control & $\boldsymbol{P}$ Value \\
\hline$<30 \mathrm{~min}$ & $96 \%$ & $40 \%$ & $<.001$ & & $40 \%$ & $4 \%$ & $<.001$ \\
$<1 \mathrm{hr}$ & $100 \%$ & $90 \%$ & .057 & & $100 \%$ & $78 \%$ & $<.001$ \\
$>1 \mathrm{hr}$ & $0 \%$ & $10 \%$ & .057 & $0 \%$ & $18 \%$ & $<.001$ \\
\hline
\end{tabular}

the scene and control of patient care defaults to the conventional EMS provider. On alternating weeks, the MSU is used in a neighboring metropolitan area.

Institutional review board permission for this study was obtained. Consecutive patients identified as prehospital stroke alerts during weeks when the MSU was operating locally from the prospectively maintained stroke alert data base were included. Control stroke-alert patients presenting from the same geographic region during weeks that the MSU was not running locally were selected from the data base on the basis of prehospital stroke-alert status and were matched for day of the week and time of day.

Time from the EMS dispatch to time of image availability in a PACS (PACS time) and time from EMS dispatch to time of a radiologist's verbal communication of results to the stroke neurologist (report time) were compared between the MSU and control patient groups. The difference between PACS time and report time represents the time during which the radiologist opens and interprets the CT scan and verbally communicates the findings via the dedicated physician-to-physician communication service (the "DocLine") of the hospital. Descriptive statistics between MSU and control patients were compared using a Student $t$ test. Categoric group differences in meeting critical benchmarks were compared using a Fisher exact test.

\section{RESULTS}

Ninety-seven patients from January 2016 through September 2016 were included for analysis, including 47 consecutive patients imaged via the MSU and 50 control patients imaged via the conventional in-hospital pathway. Mean times of dispatch to images viewable on the PACS were 21 minutes and 44 minutes in MSU and control groups, respectively $(P<.001)$. Mean times of dispatch to the radiology report were 34 minutes and 54 minutes, respectively $(P<.001)$ (Table 1$)$. SDs for both image and report times were smaller in the MSU group compared with controls.

Other key time metrics were also significantly shorter in the MSU group (Table 2). In the MSU group, images were visible on the PACS within 30 and 60 minutes in $98 \%$ and $100 \%$ of cases, respectively, compared with $40 \%$ and $90 \%$ of cases, respectively, in the control group. Similarly, in the MSU group, radiologists' reports were given to the stroke neurologist within 30 minutes and 60 minutes in $40 \%$ and $100 \%$ of cases, respectively, compared with only $4 \%$ and $78 \%$ in the control group. The MSU pathway was significantly more likely to get images to the PACS within 30 minutes and to provide a report within 60 minutes of dispatch compared with controls $(P<.001)$. Mean differences between PACS time and report time between the MSU and control groups, 13 and 11 minutes, respectively, were similar. It is not surprising that CT interpretation and reporting are streamlined in both MSU and conventional stroke-alert pathways.

\section{DISCUSSION}

This study demonstrates the considerable impact that the MSU can have on key imaging metrics within the stroke-alert treatment pathway, and it is the first to demonstrate this impact in a US market. Walter et $\mathrm{al}^{5}$ performed a similar study in Homburg, Germany, also with week-on and week-off pseudorandomization. ${ }^{5}$ That study showed a reduction of PACS time by 41 minutes from 97 to 56 minutes. In both studies, the PACS time was reduced by roughly half ( $43 \%$ in the Homburg study and 52\% in our study). The MSU facilitated provision of actionable reports within 60 minutes of dispatch, the so-called "golden hour," in $100 \%$ of patients compared with only $78 \%$ in the control group. Tighter SDs of image and report times in the MSU group suggest decreased variability and greater reliability of imaging and reporting within a given timeframe. Anecdotally, earlier CT results and examination by a neurologist facilitated by the MSU have been associated with commensurate improvements in the timeliness of tPA administration. However, the data describing the impact of the MSU on tPA utilization, expediency, and clinical outcomes are currently being collected and will be addressed in subsequent studies.

The mobile stroke unit may represent a paradigm shift in the treatment pathway for acute stroke and presents an opportunity to consider new ways in which health care can be delivered. There may be an even greater impact as the MSU is deployed to more widely distributed geographic regions. Patients from rural communities face distinct limitations and challenges in acute ischemic stroke care, some of which have been addressed by novel telestroke services. ${ }^{6,7}$ Patients from these communities not only contend with the decreased effectiveness of thrombolytic therapy resulting from delayed tPA administration secondary to the inherently longer travel times, but rural patients are also more often excluded from thrombolytic therapy entirely due to this delay. The MSU, it is hoped, should result in fewer patients being excluded from thrombolytic treatment due to time constraints. Thus, the MSU may represent an important step toward a more equitable distribution of health care delivery services across different demographic populations.

\section{CONCLUSIONS}

This study demonstrates the ability of a mobile stroke unit system to significantly reduce the time elapsed from EMS dispatch to the availability of CT acquisition and reporting. The MSU also significantly impacted the ability of a neurologist to receive an actionable radiology report within 60 minutes of dispatch. We expect that commensurate improvements in tPA delivery times and, ultimately, patient outcomes will be borne out in subsequent studies. Further additional questions will include the economic impact and cost effectiveness of the MSU in reducing the considerable cost that ischemic stroke inflicts on society. 
Disclosures: William J. Jones—RELATED: Grant: Benefits of Stroke Treatment Delivered using a Mobile Stroke Unit, Comments: Patient-Centered Outcomes Research Institute-funded, multicenter grant to study the effectiveness, including cost-effectiveness, of Mobile Stroke Units. Also, as site Principal Investigator for the BEST-MSU study, I, as well as the institution, get financial support from the BEST-MSU study*; Fees for Participation in Review Activities such as Data Monitoring Boards, Statistical Analysis, Endpoint Committees, and the Like: VOYAGER PAD, Comments: Independent Stroke Outcome Adjudicator for the Bayer Pharmaceuticals-sponsored VOYAGER PAD study. *Money paid to the institution.

\section{REFERENCES}

1. Mozzafarian D, Benjamin EJ, Go AS, et al; Writing Group Members; American Heart Association Statistics Committee, Stroke Statistics Subcommittee. On behalf of the American Heart Association Statistics Committee and Stroke Statistics Subcommittee. Heart disease and stroke statistics-2016 update: a report from the American Heart Association. Circulation 2016;133:e38-360 CrossRef Medline

2. Lees KR, Bluhmki E, von Kummer R, et al; ECASS, ATLANTIS, NINDS and EPITHET rt-PA Study Group. Time to treatment with intrave- nous alteplase and outcome in stroke: an updated pooled analysis of ECASS, ATLANTIS, NINDS, and EPITHET trials. Lancet 2010;375: 1695-703 CrossRef Medline

3. Mazighi M, Chaudhry SA, Ribo M, et al. Impact of onset-to-reperfusion time on stroke mortality: a collaborative pooled analysis. Circulation 2013;127:1980-85 CrossRef Medline

4. Cheng NT, Kim AS. Intravenous thrombolysis for acute ischemic stroke within 3 hours versus between 3 and 4.5 hours of symptom onset. Neurohospitalist 2015;5:101-09 CrossRef Medline

5. Walter S, Kostopoulos P, Haass A, et al. Diagnosis and treatment of patients with stroke in a mobile stroke unit versus in hospital: a randomised controlled trial. Lancet Neurol 2012;11:397-404 CrossRef Medline

6. Hess DC, Wang S, Hamilton W, et al. REACH: clinical feasibility of a rural telestroke network. Stroke 2005;36:2018-20 CrossRef Medline

7. Kulcsar M, Gilchrist S, George MG. Improving stroke outcomes in rural areas through telestroke programs: an examination of barriers, facilitators, and state policies. Telemed J E Health 2014;20:3-10 CrossRef Medline 